\title{
Formal Models for Context Aware Computing
}

\author{
Pooja Mohan \\ Department of IT, GGDSD College \\ Panjab University, \\ Chandigarh, India
}

\author{
Manpreet Singh \\ University College of Engineering, \\ Punjabi University, \\ Patiala, India
}

\begin{abstract}
Context-aware computing refers to a general class of mobile systems that can sense their physical environment, and adapt their behavior accordingly. In this paper we seek to develop a systematic understanding of context-aware computing by constructing a formal model and notation for expressing context-aware computations. This discussion is followed by a description and comparison of current context modeling and reasoning techniques.
\end{abstract}

Keywords: Context; Computing; Context Models; Reasoning; Ontology

\section{INTRODUCTION}

Context-awareness is considered as an important ingredient of today's most ubiquitous computing applications. The behavior of these applications is mostly characterized by embedding the interpretation logic of contextual information inside applications, creating problems for reusability of this information by other applications. Since ubiquitous computing is about interactive and smart environments, in order to enable such interactions, applications need a shared understanding of context to communicate and transfer contextual information effectively among them.

This study addresses the issue of how to represent and manage Context information. One approach is to model context using existing data modeling techniques from the field of information systems, and to store and manage the information using a database management system. It can be modeled by using both the EntityRelationship model and the class diagrams of UML. According to study [1], UML constructs are more expressive than those provided by ER, but also correspondingly more cumbersome. It provides a graphical notation for modeling concepts in order to allow context models to be specified diagrammatically. This notation takes the form of a directed graph, in which entity and attribute types form the nodes, and associations are modeled as arcs connecting these nodes. Both the context toolkit [2] and the sensor architecture of Schmidt et al. [3] support the acquisition of context data from sensors, and the processing of this raw data to obtain high-level context information. The former is a programming toolkit that can be connected together to gather and process context information from sensors. The latter provides a layered model of context processing in which sensor output is transformed into one or more cues, which undergo processing to form an abstract context description comprising a set of values, each associated with a certainty measure that estimates the certainty that the value is correct.

Chan et al. [4] model (context-based) events and event's notifications in their context-aware middleware. Harper [5] proposes a context model for a web browsing; the model includes a web-specific hypertext containing user-specific context information presented within the page.

Semantics plays an important role in the interpretation of context and its changes as driving forces behind context-aware system's actions capturing the semantics of context is primarily done by using ontologies, i.e., particular models representing the nature and relationships of context. Bouquet et al. [6] propose a model for ontologies in context-aware systems. They express the model in the Web Ontology Language (OWL, [7]). Maamar and Narendra [8] takes the use of OWL further by proposing ontology-based context resolving techniques for composing of web services. Khedr and Karmouch [9] and van Kranenburg et al. [10] propose representation of context with the use of context foundational, core and application ontologies. Moreover, Guiling et al. [11] propose a generic ontology-based model for context query, matching and context-based policies.

Concerning semantics and context-awareness, Strang and Linnhoff-Popien [12] indicate the set of challenges, like distributed composition, partial validation, richness of quality of context information, incompleteness and ambiguity, to be tackled when modeling context. They categorize context models as a key-value, markup scheme-based, graphical (e.g. Unified Modeling Language), object-oriented, logic- and ontology-based. 
They indicate the latter models as the most promising for the future.

Similarly, Razzaque et al. [13] indicate set-theory-based, directedgraph based and first-order-logic based context models, emphasizing the necessity of modeling user's preferences and profiles in comprehensive data structures exposing the dependency relations between the user's preferences and profiles.

\section{CHARACTERISTICS OF CONTEXT INFORMATION}

Several requirements have to be taken into account when modeling context information [14]:

2.1 Heterogeneity and mobility: Context data obtained from databases or digital libraries - like geographic map data-are often static. Many context-aware applications are also mobile or depend on mobile context information sources. This adds to the problem of heterogeneity as the context information provisioning must be adaptable to the changing environment. Also, location and spatial layout of the context information play important roles due to this requirement.

2.2 Relationships and dependencies: There exist various relationships between types of context information that have to be captured to ensure correct behavior of the applications. One such relationship is dependency whereby context information entities/facts may depend on other context information entities.

2.3 Timeliness: Context-aware applications may need access to past states and future states (prognosis). Therefore, timeliness (context histories) is another feature of context information that needs to be captured by context models.

2.4 Imperfection: Due to its dynamic and heterogeneous nature, context information may be of variable quality. In fact, it may even be incorrect. Most sensors feature an inherent inaccuracy and the sensed values age if the physical world changes, so that this inaccuracy increases over time. Thus, a good context modeling approach must take these problems into account to enable proper reasoning about context information changes to achieve appropriate adaptations for the application, and thus provide an experience for the user that is consistent with the physical world.

2.5 Reasoning: Context-aware applications use context information to evaluate whether there is a change to the user and/or to the environment situation; taking a decision whether any adaptation to that change is necessary often requires reasoning capabilities. Reasoning techniques can also be adopted to derive higher level context information.

\section{CONTEXT MODELING APPROACHES}

\subsection{Graphical Models of context information}

A very well known general purpose modeling instrument is the Unified Modeling Language (UML) which has a strong graphical component (UML diagrams). Due to its generic structure, UML is also appropriate to model the context. This is shown for instance by Bauer in [15], where contextual aspects relevant to air traffic management are modeled as UML extensions.

Another example is the nicely designed graphics oriented context model introduced in [16] by Henricksen et al., which is a context extension to the Object-Role Modeling (ORM) approach [17] according some contextual classification and description properties. In ORM, the basic modeling concept is the fact, and the modeling of a domain using ORM involves identifying appropriate fact types and the roles that entity types play in these. Henricksen extended ORM to allow fact types to be categorized according to their persistence and source, either as static (facts that remain unchanged as long as the entities they describe persist) or as dynamic. The latter ones are further distinguished depending on the source of the facts as either profiled, sensed or derived types. Another quality indicator introduced by Henricksen is a history fact type to cover a time-aspect of the context. The last extension to ORM made by Henricksen for context modeling purposes are fact dependencies, which represent a special type of relationship between facts, where a change in one fact leads automatically to a change in another fact: the dependsOn relation. This kind of approach is particularly applicable to derive an ERmodel from it, which is very useful as structuring instrument for a relational database in information system based context management architecture such as the one described in [18].

Halpin [19] describes the Rmap procedure for transforming a conceptual schema to a relational schema, and Henricksen [20] has developed an extension of Rmap that can be used to map a CML-based context model to a relational database. However, the formal semantics of ORM and CML can be leveraged to provide integration with other implementations such as fact-based reasoners (though it should be noted that some features of CMLparticularly the constructs related to imperfect information-may not be supported). CML leverages the formality of ORM to 
support the evaluation of simple assertions as well as SQL-like queries.

\subsection{Spatial context model}

Space is an important context in many context-aware applications. Most context definitions mention space as a vital factor: e.g., Schilit, Adams and Want define three important aspects of context as "Where you are, who you are with and what resources are nearby" [21]. Thus, some context modeling approaches give space and location a preferential treatment. Most spatial context models are fact-based models that organize their context information by physical location. Spatial context models can be described along the tiers of spatial ontologies proposed by A. Frank [22]: Ontology based models of context information typically cover all 4 tiers. Although the tiered model of Frank is just an abstract conceptualization of different (spatial) representations of the world, it is useful to distinguish between various implementations of spatial context models.

The spatial context model developed in the Nexus project (called Augmented World Model [23]) is an object-based class hierarchy of context information that supports multi-inheritance. In contrast to the Nexus model, the Equator project context model [24] is a typical contextual ontology that represents all tiers by an OWL class model. Its location model is a hierarchical notion of interconnected symbolic spaces, such as Buildings, Floors and Rooms. Properties define spatial relations between these spaces. Although the ontology also offers coordinate features, Millard et al. states that it is very hard to perform any inference over them using a normal reasoner, as they are usually not spatially aware.

\subsection{Ontology based models of context information}

Ontologies are essentially descriptions of concepts and their relationships; it is not surprising that the subset of the OWL language admitting automatic reasoning (i.e., OWL-DL) is indeed description logic. The formalism of choice in ontology-based models of context information is typically OWL-DL [25] or some of its variations, since it is becoming a de-facto standard in various application domains, and it is supported by a number of reasoning services. By means of OWL-DL it is possible to model a particular domain by defining classes, individuals, characteristics of individuals (data type properties), and relations between individuals (object properties). For instance, given two atomic classes Person and Female, the class Male can be defined as: Male $\equiv$ Person $\neg$ Female

www.ijcat.com
Various OWL ontologies have been proposed for representing shared descriptions of context data. Among the most prominent proposals are the SOUPA [26] ontology for modelling context in pervasive environments, and the CONON [27] ontology for smart home environments. OWL-DL ontological models of context have been adopted in several architectures for context-awareness; among the others, we recall the Context Broker Architecture (CoBrA) [28] and the SOCAM [29] middleware, that adopt the SOUPA and CONON ontologies, respectively.

\subsection{Logic Based Models}

A logic defines the conditions on which a concluding expression or fact may be derived (a process known as reasoning or inferencing) from a set of other expressions or facts. To describe these conditions in a set of rules a formal system is applied. In a logic based context model, the context is consequently defined as facts, expressions and rules. One of the first logic based context modeling approaches has been researched and published as Formalizing Context in early 1993 by McCarthy and his group at Stanford [30, 31]. McCarthy introduced contexts as abstract mathematical entities with properties useful in artificial intelligence. He prevented emphatically to give a definition what context is. Instead he tried to give a formalization recipe which allows for simple axioms for common sense phenomena, e.g. axioms for static blocks worlds situations, to be lifted to context involving fewer assumptions, e.g. contexts in which situations change. Thus lifting rules, which relate the truth in one context to the truth in another context, are an important part of the model itself. The basic relation in this approach is $i s t(c, p)$, which asserts that the it proposition $\mathrm{p}$ is true in the context $c$. This allows for formulas such as c0: ist(contextof("Sherlock Holmes stories"), "Holmes is a detective"), where $\mathrm{c} 0$ is considered to be an outer context. A similar approach is the Sensed Context Model proposed by Gray and Salber [32]. They use first-order predicate logic as a formal representation of contextual propositions and relations. Another approach within this category is the multimedia system by Bacon et al. [33]. In this system the location as one aspect of the context is expressed as facts in a rule based system. The system itself is implemented in Prolog.

\subsection{Key Value Models}

The model of key-value pairs is the most simple data structure for modeling contextual information. Already Schilit et al. [21] used key-value pairs to model the context by providing the value of context information (e.g. location information) to an application as an environment variable. The key-value modeling approach is 
frequently used in distributed service frameworks. In such frameworks, the services itself are usually described with a list of simple attributes in a key-value manner, and the employed service discovery procedure operates an exact matching algorithm on these attributes.

\subsection{Markup Scheme Models}

Common to all markup scheme modeling approaches is a hierarchical data structure consisting of markup tags with attributes and content. In particular, the content of the markup tags is usually recursively defined by other markup tags. Typical representatives of this kind of context modeling approach are profiles. They usually base upon a serialization of a derivative of Standard Generic Markup Language (SGML), the superclass of all markup languages such as the popular XML. Some of them are defined as extension to the Composite Capabilities / Preferences Profile (CC/PP) [34] and User Agent Profile (UAProf) [35] standards, which have the expressiveness reachable by RDF/S and a XML serialization. An example of this approach is the Comprehensive Structured Context Profiles (CSCP) by Held et al. [36]. Drawback of CC/PP, the restricted overriding mechanism of default values only, replaced by a more flexible overriding and merging mechanism, allowing for instance to override and/or merge a whole profile subtree. A similar approach to CSCP is the CC/PP Context Extension by Indulska et al. [37]. They extended the basic CC/PP and UAProf vocabulary by a number of component-attribute trees related to some aspects of context, e.g. concerning location, network characteristics, application requirements, session information as well as certain types of relations and dependencies. Another context modeling approach in the markup scheme category - which does not bear towards $\mathrm{CC} / \mathrm{PP}$ - is the Pervasive Profile Description Language (PPDL) [38]. This XMLbased language allows accounting for contextual information and dependencies when defining interaction patterns on a limited scale. There are several other context modeling approaches in the markup scheme category. They are oftentimes either proprietary or limited to a small set of contextual aspects, or both. Examples affected by these limitations are, among others, the context configuration of Capra et al.'s reflective middleware [39] the Centaurus Capability Markup Language (CCML) [40], ConteXtML [41].

\subsection{Object Oriented Models}

Common to object oriented context modeling approaches is the intention to employ the main benefits of any object oriented approach - namely encapsulation and reusability - to cover parts of the problems arising from the dynamics of the context in ubiquitous environments. An approach within the object category is the Active Object Model of the GUIDE project [42]. Again, the chosen approach has been primarily driven by the requirement of being able to manage a great variety of personal and environmental contextual information while maintaining scalability. All the details of data collection and fusing (e.g. the context adaptive composition of HTML fragments) are encapsulated within the active objects and thus hidden to other components of the system.

\subsection{Hybrid models}

Henricksen et al. [43] propose a hybrid approach to context modelling, combining ontologies with the fact based approach provided by the CML language. The goal is to combine the particular advantages of CML models (especially the handling of ambiguous and imperfect context information) with interoperability support and various types of reasoning provided by ontological models. The hybrid approach is based on a mapping from CML modeling constructs to OWL-DL classes and relationships. It is worth noting that, because of some expressivity limitations of OWL-DL, a complete mapping between CML and OWL-DL cannot be obtained.

With respect to interoperability issues, the advantages gained by an ontological representation of the context model are clearly recognizable. However, with respect to the derivation of new context data, experiences with the proposed hybrid model showed that ontological reasoning with OWL-DL and its SWRL extension did not bring any advantage with respect to reasoning with the CML fact-based model. For this reason, ontological reasoning is performed only for automatically checking the consistency of the context model, and for semantic mapping of different context models. [44] Presents creation of generic context ontology, and a location-based context model. The designed ontology is called COMANTO and describes general context types and interrelationships that are not domain-, application- or situationspecific. The location-based context model proposed focuses on addressing context management challenges in distributed pervasive environments, and is integrated with the COMANTO context knowledge. The combined modeling approach aims to enable efficient management of context data and allow for widely applicable context formalism. 


\section{CONCLUSION}

In the paper we presented a set of requirements that context modeling and reasoning techniques should meet. The discussion of the requirements was followed by a description of the most prominent, approaches to context modeling. These approaches are rooted in database modeling techniques and in ontology based frameworks for knowledge representation. Spatial models provide efficient procedures for the execution of typical spatial queries; however, they do not always cope with the uncertainty of actual location readings. With regard to fact-based models, the CML language has advantages in its support for software engineering and in the good balance between expressive power and efficient reasoning procedures for that language. Indeed, the predicate logic supported by CML is well suited for expressing dynamic situations. However, in order to preserve efficiency, that language is less expressive than ontological languages like OWL-DL. Finally, ontological models have clear advantages regarding support for a) interoperability, b) heterogeneity, and c) representation of complex relationships and dependencies among context data. However, when considering the tradeoff between expressiveness and complexity, the choice of ontological models may not always be satisfactory.

\section{REFERENCES}

[1] Karen Henricksen, K., Jadwiga Indulska, J., "Modeling Context Information in Pervasive Computing Systems", 2002, pp. 167-180.

[2] Dey, A., Salber, D., Abowd, G., "A context-based infrastructure for smart environments", in: 1st International Workshop on Managing Interactions in Smart Environments (MANSE'99). (1999), pp. 1-15.

[3] Schmidt, A., et al. "Advanced interaction in context" In: 1st International Symposium on Handheld and Ubiquitous Computing (HUC'99), Karlsruhe (1999)

[4] Chan, A., et al., "An Event-Driven Middleware for Mobile Context Awareness", The Computer Journal, 2004, 47(3), pp. 278-288.

[5] Harper, S., "Middleware to Expand Context and Preview in Hypertext", In ACM SIGACCESS conference on Computers and accessibility. 2004. Atlanta, GA, USA: ACM Press.

[6] Bouquet, P., et al., "C-OWL: Contextualizing Ontologies", In Second Intl Semantic Web Conference (ISWC03). 2003. Las Vegas, Nevada, USA: Springer Verlag.

[7] Zuo, Z. and M. Zhou, "Web Ontology Language OWL and its description logic foundation", In 4th Intl Conference on Parallel and Distributed Computing, Applications and Technologies (PDCAT03). 2003: IEEE Press.

[8] Maamar, Z. and N.C. Narendra, "Ontology-based Context www.ijcat.com
Reconciliation in a Web Services Environment: From OWL$S$ to OWL-C", In Workshop on Web Services and Agentbased Engineering (WSABE). 2004. New York City, USA.

[9] Khedr, M. and A. Karmouch, "ACAI: Agent-Based Contextaware Infrastructure for Spontaneous Applications", Journal of Network \& Computer Applications, 2005. 28(1): pp. 1944

[10] Kranenburg, H., et al., "Grounded Contextual Reasoning enabling Innovative Mobile Services", In 5th Workshop on Applications and Services in Wireless Networks (ASWN05). 2005. Grenoble, France, pp. 93-102

[11] Guiling, W., J. Jinlei, and S. Meilin, “ A Context Model for Collaborative Environment". In 10th Intl. Conference on Computer Supported Cooperative Work in Design. 2006: IEEE Press., pp. 1-6

[12] Strang, T. and C. Linnhoff-Popien., "A Context Modeling Survey", In UbiComp 1st Intl Workshop on Advanced Context Modeling, Reasoning and Management. 2004.

[13] Razzaque, M.A., S. Dobson, and P. Nixon., "Categorization and Modeling of Quality in Context Information", In Workshop on AI and Autonomic Communications (IJCAI05). 2005.

[14] Shehzad, A., Hung Q. Ngo, Kim Anh Pham, and S. Y. Lee, "Formal Modeling in Context Aware Systems", http://citeseerx.ist.psu.edu/viewdoc/summary?doi=10.1.1.13 1.1231

[15] Bauer, J., "Identification and Modeling of Contexts for Different Information Scenarios in Air Traffic", Mar. 2003. Diplomarbeit.

[16] Henricksen, K., Indulska, J., and Rakotonirainy, A. "Generating Context Management Infrastructure from HighLevel Context Models", In Industrial Track Proceedings of the 4th International Conference on Mobile Data Management (MDM2003) (Melbourne/Australia, January 2003), pp. 1-6.

[17] Halpin, T. A., "Information Modeling and Relational Databases: From Conceptual Analysis to Logical Design", Morgan Kaufman Publishers, San Francisco, 2001.

[18] Indulska, J., Robinsona, R., Rakotonirainy, A., and Henricksen, K., "Experiences in using cc/pp in contextaware systems", In LNCS 2574: Proceedings of the 4th International Conference on Mobile Data Management (MDM2003) (Melbourne/Australia, January 2003), M.-S. Chen, P. K. Chrysanthis, M. Sloman, and A. Zaslavsky, Eds., Lecture Notes in Computer Science (LNCS), Springer, pp. 247-261.

[19] Halpin, T. A., "Conceptual Schema and Relational Database Design”, 2nd ed., Prentice Hall Australia, Sydney, 1995.

[20] Henricksen, K., "A framework for context-aware pervasive computing applications", Ph.D. thesis, School of Information Technology and Electrical Engineering, The University of Queensland (September 2003).

[21] Schilit, B, Adams, N., R. Want, et al., "Context-aware Computing Applications", Xerox Corp., Palo Alto Research Center, 1994.

[22] Frank, A., "Tiers of ontology and consistency constraints in geographical information systems", International Journal of Geographical Information Science 15 (7) (2001), pp. 667678. 
[23] Nicklas, D., Mitschang, B., "The Nexus Augmented World Model: An extensible approach for mobile, spatially aware applications", 7th International Conference on ObjectOriented Information Systems.2001, pp. 392-401

[24] Millard, I., D. De Roure, Shadbolt, N., "The use of ontologies in contextually aware environments", In Proceedings of First International Workshop on Advanced Context (2004), pp. 42-47.

[25] Horrocks, I., Patel-Schneider, P.F., F. van Harmelen, "From SHIQ and RDF to OWL: The making of a web ontology language", Journal of Web Semantics 1 (1) (2003), pp. 7-26.

[26] H. Chen, F. Perich, T. W. Finin, A. Joshi, "SOUPA: Standard Ontology for Ubiquitous and Pervasive Applications", in: 1st Annual International Conference on Mobile and Ubiquitous Systems (MobiQuitous 2004), IEEE Computer Society, 2004.

[27] D. Zhang, T. Gu, X.Wang, "Enabling Context-aware Smart Home with Semantic Technology", International Journal of Human-friendly Welfare Robotic Systems 6 (4) (2005), pp. $12-20$.

[28] Chen, H., Finin, T., Joshi, A., "Semantic Web in the Context Broker Architecture", in: Proceedings of the Second IEEE International Conference on Pervasive Computing and Communications (PerCom 2004), IEEE Computer Society, 2004.

[29] Gu, T., Wang, H. K. , Pung, H.K., Zhang, D.Q., "An ontology-based context model in intelligent environments", in: Proceedings of Communication Networks and Distributed Systems Modeling and Simulation Conference, San Diego, California, USA, 2004.

[30] McCarthy, J., "Notes on formalizing contexts", In Proceedings of the Thirteenth International Joint Conference on Artificial Intelligence (San Mateo, California, 1993), R. Bajcsy, Ed., Morgan Kaufmann, pp. 555-560.

[31] Mccarthy, J., and Buva., "Formalizing context". In Working Papers of the AAAI Fall Symposium on Context in Knowledge Representation and Natural Language (Menlo Park, California, 1997), American Association for Artificial Intelligence, pp. 99-135.

[32] Gray, P., Salber, D., "Modelling and Using Sensed Context Information in the design of Interactive Applications", In LNCS 2254: Proceedings of 8th IFIP International Conference on Engineering for Human-Computer Interaction (EHCI 2001), May 2001, M. R. Little and L. Nigay, Eds., Lecture Notes in Computer Science (LNCS), Springer, p. $317 \mathrm{ff}$.

[33] Bacon, J., Bates, J., Halls, D., "Location-oriented multimedia”, IEEE Personal Communications 4, 5 (1997).

[34] W3C. Composite Capabilities / Preferences Profile (CC/PP). http://www.w3.org/Mo- bile/CCPP.
[35] Wapforum. User Agent Profile (UAProf). http://www.wapforum.org.

[36] Held, A., Buchholz, S., Sachill, A., "Modeling of context information for pervasive computing applications", In Proceedings of SCI 2002/ISAS 2002 (2002).

[37] Indulska, J., Robinsona, R., "Experiences in using cc/pp in context-aware systems", In LNCS 2574: Proceedings of the 4th International Conference on Mobile Data Management (MDM2003) (Melbourne/Australia, January 2003), M.-S Chen,P. K. Chrysanthis, M. Sloman, and A. Zaslavsky, Eds.,Lecture Notes in Computer Science (LNCS), Springer, pp. 247-261.

[38] Chtcherbina, E., Franz, M., "Peer-to-peer coordination framework (p2pc): Enabler of mobile ad-hoc networking for medicine, business, and entertainment", In Proceedings of InternationalConference on Advances in Infrastructure for Electronic Business, Education, Science, Medicine, and Mobile Technologies on the Internet (SSGRR2003w) (L'Aquila/Italy, January 2003).

[39] Capra, L., Emmerich, W., Mascolo, C., "Reflective middleware solutions for context-aware applications", 2001.

[40] Kagal, L., Korolev, V., Chen, H., Joshi, A., Finin, T., "Project centaurus: A framework for indoor mobile services".

[41] Ryan, N., "ConteXtML: Exchanging Contextual Information between a Mobile Client and the FieldNote Server", August 1999.

[42] Cheverst, K., Mitchell, K., Davies, N., "Design of an object model for a context sensitive tourist GUIDE", Computers and Graphics 23, 6 (1999), pp. 883-891.

[43] Henricksen, K., Livingstone, S., Indulska, J., "Towards a Hybrid Approach to Context Modeling, Reasoning and Interoperation", in: J. Indulska, D. D. Roure (eds.), Proceedings of the First International Workshop on Advanced Context Modelling, Reasoning And Management, in conjunction with UbiComp 2004, Nottingham, England: University of Southhampton, 2004.

[44] Roussaki, I., Strimpakou, M. et al, "Hybrid context modeling: A location-based scheme using ontologies," percomw, pp.2-7, Fourth IEEE International Conference on Pervasive Computing and Communications Workshops (PERCOMW'06), 2006 